\title{
Reciprocal duplication of the Williams-Beuren syndrome deletion on chromosome $7 q 11.23$ is associated with schizophrenia
}

\author{
Jennifer Gladys Mulle ${ }^{a, b}$, Ann E. Pulver ${ }^{c, d}$, John M. McGrath ${ }^{c}$, Paula Wolyniec ${ }^{c}$, Anne F. \\ Dodd $^{a}$, David J. Cutlerb, Jonathan Sebate,f,g,h, Dheeraj Malhotra ${ }^{e, f}$, Gerald Nestadt ${ }^{c}$, Donald \\ F. Conradi, Matthew Hurlesi, Chris P. Barnes', Masashi Ikedak, Nakao Iwatak, Douglas F. \\ Levinson', Pablo V. Gejman ${ }^{m, n}$, Alan R. Sanders ${ }^{m, n}$, Jubao Duan ${ }^{m, n}$, Adele A. Mitchell ${ }^{\circ}$, Inga \\ Peter $^{p}$, Pamela Sklar ${ }^{q, r, s}$, Colm T. O’Dushlaine ${ }^{q, r, t}$, Detelina Grozeva ${ }^{\mathrm{u}}$, Michael C. \\ O’Donovan ${ }^{\mathrm{u}}$, Michael J. Owen ${ }^{\mathrm{u}}$, Christina M. Hultman ${ }^{v}$, Anna K. Kähler ${ }^{\mathrm{v}, w, x}$, Patrick F. \\ Sullivan $^{w}$, George Kirov ${ }^{u}$, and Stephen T. Warren ${ }^{b, y}$ \\ ${ }^{a}$ Department of Epidemiology, Rollins School of Public Health, Emory University, Atlanta, \\ Georgia, USA \\ ${ }^{b}$ Department of Human Genetics, Emory University School of Medicine, Atlanta Georgia, USA \\ 'Department of Psychiatry and Behavioral Sciences, Johns Hopkins School of Medicine, \\ Baltimore, Maryland, USA \\ dDepartment of Epidemiology, Johns Hopkins Bloomberg School of Public Health, Baltimore, \\ Maryland, USA \\ eBeyster Center for Genomics of Psychiatric Diseases, University of California, San Diego, La \\ Jolla, California, USA \\ fDepartment of Psychiatry, University of California, San Diego, La Jolla, California, USA \\ gDepartment of Cellular Molecular and Molecular Medicine, University of California, San Diego, \\ La Jolla, California, USA \\ hInstitute for Genomic Medicine, University of California, San Diego, La Jolla, California, USA \\ iThe Wellcome Trust Sanger Institute, Wellcome Trust Genome Campus, Hinxton, Cambridge, \\ UK \\ iDepartment of Cell and Developmental Biology, University College London, London, UK
}

\footnotetext{
(C) 2013 Society of Biological Psychiatry. Published by Elsevier Inc. All rights reserved.

Corresponding author: Jennifer Gladys Mulle, MHS, PhD, Rollins Assistant Professor, Rollins School of Public Health, Emory University, Atlanta, GA 30322, (404) 727-3042 (phone), jmulle@emory.edu.

Financial Disclosures:

Dr. Mulle reports a consultancy with the Centers for Disease Control. Profs Owen, O’Donovan, and Kirov have received research funding from the Wellcome Trust and the Medical Research Council, London, UK. The Bulgarian Trios were recruited with a grant from the Janssen Research Foundation, Beerse, Belgium. Dr Sullivan was a member of the SAB of Expression Analysis (Durham, $\mathrm{NC})$.

Co-authors Pulver, McGrath, Wolyniec, Dodd, Cutler, Sebat, Malhotra, Nestadt, Conrad, Hurles, Barnes, Ikeda, Iwata, Levinson, Gejman, Sanders, Duan, Mitchell, Peter, Sklar, O'Dushlaine, Grozeva, Hultman, Kähler, and Warren reported no biomedical financial interests or potential conflicts of interest.

Publisher's Disclaimer: This is a PDF file of an unedited manuscript that has been accepted for publication. As a service to our customers we are providing this early version of the manuscript. The manuscript will undergo copyediting, typesetting, and review of the resulting proof before it is published in its final citable form. Please note that during the production process errors may be discovered which could affect the content, and all legal disclaimers that apply to the journal pertain.
} 
kFujita Health University School of Medicine, Toyake, Aichi, Japan

'Department of Psychiatry and Behavioral Sciences, Stanford University, Stanford, California, USA

mDepartment of Psychiatry and Behavioral Sciences, NorthShore University HealthSystem, Evanston, Illinois, USA

nDepartment of Psychiatry and Behavioral Sciences, University of Chicago, Chicago, Illinois, USA 'Department of Forensic Biology, Office of Chief Medical Examiner of the City of New York, $421 \mathrm{E}$ 26th Street, New York, New York, USA

PDepartment of Genetics and Genomic Sciences, Mount Sinai School of Medicine, New York, New York, USA

qPsychiatric and Neurodevelopmental Genetics Unit, Massachusetts General Hospital, Boston, MA

'Stanley Center for Psychiatric Research, Broad Institute of Harvard and MIT, Cambridge, MA

sDivision of Psychiatric Genomics, Department of Psychiatry, Mount Sinai School of Medicine, NY, NY

tAnalytic and Translational Genetics Unit, Massachusetts General Hospital, Boston, MA

uDepartment of Psychological Medicine, Cardiff University, Henry Wellcome Building, Heath Park, Cardiff CF14 4XN, UK

vDepartment of Medical Epidemiology and Biostatistics, Karolinska Institutet, Stockholm, Sweden

wDepartments of Genetics and Psychiatry, University of North Carolina, Chapel Hill, North Carolina, USA

xDivision of Mental Health and Addiction, Oslo University Hospital, Oslo, Norway

yepartments of Biochemistry and Pediatrics, Emory University School of Medicine, Atlanta, Georgia, USA

\section{Abstract}

Background-Several copy number variants (CNVs) have been implicated as susceptibility factors for schizophrenia (SZ). Some of these same CNV also increase risk for autism spectrum disorders (ASD), suggesting an etiologic overlap between these conditions. Recently, de novo duplications of a region on chromosome 7q11.23 were associated with ASD. The reciprocal deletion of this region causes Williams-Beuren syndrome (WBS).

Methods-We assayed an Ashkenazi Jewish cohort of 554 SZ cases and 1014 controls for copy number variation $(\mathrm{CNV})$, using a high-density genome-wide array. An excess of large rare and de novo $\mathrm{CNV}$ were observed, including a $1.4 \mathrm{Mb}$ duplication on chromosome 7q11.23 identified in two unrelated patients. To test whether this 7q11.23 duplication is also associated with SZ, we obtained data for 14,387 SZ cases and 28,139 controls from seven additional studies with highresolution genome-wide CNV detection. We performed a meta-analysis, correcting for study population of origin, to assess whether the 7q11.23 duplication is associated with SZ.

Results-We find duplications at 7q11.23 in 11 of 14,387 SZ cases with only 1 in 28,139 controls (unadjusted odds ratio, 21.52 , $95 \%$ CI: 3.13-922.6, p-value $5.5 \times 10^{-5}$; adjusted odds ratio 10.8, 95\% CI: 1.46-79.62, p-value 0.007). Of three SZ duplication carriers with available detailed retrospective data, all show social anxiety and language delay premorbid to SZ onset, consistent with both human studies and animal models of the 7q11.23 duplication. 
Conclusion-We have identified a new CNV associated with SZ. Reciprocal duplication of the Williams syndrome deletion at chromosome 7q11.23 confers an approximately 10-fold increase in risk for SZ.

\section{Keywords}

Schizophrenia; 7q11.23 duplication syndrome; Psychiatric genetics; Schizophrenia genetics; Autism; Williams-Beuren syndrome

\section{Introduction}

Schizophrenia (SZ) is a severe psychiatric disorder that represents a significant public health burden, affecting $1 \%$ of the population worldwide(1). It has long been recognized that genetic factors must play a role in susceptibility, but until recently identification of such risk factors has proven elusive(1). Landmark studies of genomic structural variation in SZ, first published in 2008, established that there is an excess of large, rare copy number variants (CNVs) in SZ populations(2-5). Since then, this has become one of the most exciting and consistently replicated findings in psychiatric genetics. Currently at least ten specific CNV loci have been replicated in multiple reports and can thus be regarded as confirmed risk factors (3, 5-14). The first of these reports include two large studies with thousands of patients, where recurrent deletions were found at three new loci: 1q21, 15q11 and 15q13, in addition to the previously identified $22 \mathrm{q} 11$ deletion $(5,15)$. Several studies soon followed, implicating deletions at 17p12(16) and 3q29(7, 8), and duplications at 16p11(12). These variants have the following properties in common: they are all large, involving many genes; they are rare, at a frequency far less than $1 \%$ even in SZ populations; and they all carry substantial risk for SZ, with estimated odds ratios between 3-30(7, 14). Smaller variants have been identified in specific genes, including deletions in neurexin1 (NRXN1)(9, 10, 17) and contactin-associated protein-like 2 (CNTNAP2), another member of the neurexin family. (11). Duplication at the vasoactive intestinal peptide receptor 2 (VIPR2) locus is also associated with high risk for schizophrenia $(7,13)$. One surprising finding is that many of the $\mathrm{CNVs}$ associated with $\mathrm{SZ}$ are also significantly more common in individuals with intellectual disability (ID), autism, and epilepsy(16, 18-21), advancing the concept of a neurodevelopmental link between these disorders(22).

In the current study we initially assessed a sample of 554 independent SZ cases and 1014 controls, all of Ashkenazi Jewish (AJ) descent. We limited our analysis to large rare CNVs in keeping with previous reports. We replicate previous findings of rare and de novo CNVs, finding a significant excess of these events in SZ cases compared to controls. Among these rare events, we discovered two duplications on chromosome $7 \mathrm{q} 11.23$, including one confirmed de novo event. A subsequent meta-analysis of 14,387 SZ cases and 28,139 controls confirmed the overrepresentation of this duplication in SZ cases, with an estimated odds ratio of 10.8 (95\% CI: 1.46-79.62, p-value 0.007 ). De novo duplications at this identical locus were previously reported in an autism population(23), further supporting a shared etiology between autism and schizophrenia.

\section{Methods and Materials}

\section{Study subjects}

SZ Cases-Ashkenazi Jewish individuals affected with SZ ( $n=615)$ were recruited nationally over a six-year period. Cases were eligible for inclusion in these analyses if the proband met DSM-IV criteria for a SZ diagnosis and all four grandparents were of Ashkenazi Jewish descent. When available, parental DNAs were also collected. Probands were assessed for psychiatric illness according to an established consensus-based procedure, 
as described in(24) and in the Supplement. No subject in our study had a previous clinical genetic diagnosis.

Controls-Control subjects were selected from three cohorts: a study of Crohn's disease in the Ashkenazim ( $\mathrm{n}=258)$, a study of neuromuscular disease (Parkinson's and dystonia) in the Ashkenazim ( $\mathrm{n}=266)$, and the Ashkenazi Jewish Control Registry (AJCR) hosted at Johns Hopkins University ( $\mathrm{n}=538$ ). Control subjects from the Crohn's and neuromuscular cohorts were not screened for psychiatric disease; AJCR controls were administered a questionnaire about psychiatric conditions.

Meta-Analysis-Seven additional samples were incorporated into our meta-analysis, ultimately totaling 14,387 SZ cases and 28,139 unaffected controls. Details of each sample, including ascertainment criteria and genotyping platform, are included in the Supplement.

For the Ashkenazi Jewish sample ascertained at Johns Hopkins, all recruitment methods and protocols for collection of clinical data and blood samples were approved by the Johns Hopkins institutional review board, and informed consent was obtained from all individuals. All other data, including data for the meta-analysis, were fully anonymized prior to receipt at Emory University.

Genotyping and identification of CNVs-DNA from the Ashkenazi sample was extracted using the Gentra Puregene kit at Johns Hopkins University. All DNA used for this study was extracted from blood (no cell line DNA was used). Genotyping was performed using the Affymetrix Human Genome-Wide SNP Array 6.0 at Emory University. Genotypes were called using the Birdseed algorithm, as implemented in Affymetrix power tool software (version 1.12.0). SNPs with completion rates $<90 \%$ were excluded, rendering 816,284 autosomal SNPs for analysis. For CNV analysis, normalization and log ratio data calculation was obtained using the Affymetrix power tools software (version 1.12.0). $\log (2)$ ratio data for autosomes were extracted and analyzed using three algorithms: $\operatorname{GLAD}(25)$, GADA(26), and BEAST(27). CNVs called by only a single algorithm were removed from analysis. Putative CNV intervals were filtered by size (>100 kb), number of SNPs in the CNV interval (> $20 \mathrm{SNPs}$ ), and CNV-interval SNP homozygosity rates. CNVs $>500 \mathrm{~kb}$ were validated by a second array (Illumina Human OmniExpress v1 genotyping array), qPCR, or PCR across deletion breakpoint. See Methods in the Supplement for specific details on validation methodologies.

Definition of "rare CNV"-We filtered variants with the goal of excluding typical polymorphic events. To facilitate comparison with our earlier study(8), we excluded variants that had $>50 \%$ overlap with a CNV in the database of genomic variants (DGV, http:// projects.tcag.ca/variation/, Nov 02, 2010 update) for deletions and duplications separately. We also excluded any variant with a frequency $>0.5 \%$ at any single locus.

Statistical analysis-Permutation-corrected p-values for overall CNV burden were calculated using PLINK. All odds ratios, associated confidence intervals, and p-values were calculated using the R statistical software package. Meta-Analysis statistics were calculated with the Cochran-Mantel-Haenszel exact test, stratified by study, in the R statistical package $(28,29)$. 


\section{Results}

\section{Rare and de novo CNV in the AJ population}

There was a 1.29-fold excess of rare CNVs > $100 \mathrm{~kb}$ (see Supplement), which became more pronounced for $\mathrm{CNV}>500 \mathrm{~kb}$. In the $554 \mathrm{AJ} \mathrm{SZ}$ cases compared to 1014 controls, $5.6 \%$ of cases vs. $2.4 \%$ of controls had at least one rare CNV > $500 \mathrm{~kb}$ (OR: 2.44, P-value 0.001), consistent with previous reports(2-5). The OR is strongest for deletions $>1 \mathrm{Mb}(\mathrm{OR} 5.1$, pvalue 0.004 ) (Table 1). These data harbor known susceptibility variants, including three 22q11 deletions and four 16p11 duplications, which are now considered established risk factors for SZ (see data and Table S4 in the Supplement). For 292 of these SZ cases, both parental DNAs are available, enabling us to estimate the de novo rate for large (>500 kb) rare $\mathrm{CNV}$ in our sample. We find 6 de novo events, or a rate of $2.1 \%$. We compared this to the de novo rate in non-psychiatric trios, reviewed by Kirov et al (2012)(30), where a rate of $0.8 \%$ was reported ( 27 de novo $\mathrm{CNV}$ in 3495 trios). This difference is statistically significant (OR: 2.69, p-value 0.038). Our de novo rate of $2.1 \%$ is identical to that reported in SZ (2.1\% in the study by Kirov et al (2012)(30)) and lower, but not significantly so, than the reported de novo rate in simplex ASD trios(23), (2.1\% vs 3.9\%, p-value 0.13$)$.

\section{Meta-Analysis of the $7 q 11.23$ region}

The six de novo $\mathrm{CNV}>500 \mathrm{~kb}$ in the $\mathrm{AJ}$ sample included one $1.3 \mathrm{Mb}$ de novo duplication on chromosome 7q11.23. A duplication at this locus was also identified in an additional unrelated proband (parental DNAs were unavailable for inheritance testing) (Figure 1). Given the recurrent nature of this duplication, and its involvement in ASD, we were motivated to investigate its frequency in additional SZ cohorts. This was the only variant tested in additional samples. We analyzed datasets where we could obtain the raw intensity files, to enable consistent data processing (Table S3 in the Supplement). In total, we found 11 duplications in 14,387 cases $(0.076 \%)$ and one in 28,139 controls $(0.0035 \%)$ (Table 2 and Figure 2), for an overall odds ratio of 21.5 (95\% CI: 3.13-922.6, p-value 5.5 $\times 10^{-5}$ ). Although such a large CNV would be found with any of the array platforms used and there is no reason to suspect population differences for a CNV with such a high pathogenic effect and de novo rate, we nevertheless also calculated the Mantel-Haenszel corrected OR for this duplication, leading to a corrected OR of 10.8 (p-value 0.007). These data therefore indicate the 7q11.23 duplication is a significant new risk factor for SZ.

\section{Discussion}

We have identified a new risk factor for schizophrenia, a 1.4 Mb duplication on chromosome 7q11.23. Two prior studies reported single 7q11.23 duplication carriers in their $\mathrm{SZ}$ case populations patients $(30,31)$. In this current study we find the duplication in $0.076 \%$ of SZ patients and establish a statistically significant enrichment compared to controls. The 7q11.23 duplication syndrome has previously been described (OMIM \#609757) in the context of intellectual disability and autism $(23,32,33)$. It is found in $0.1-0.12 \%$ (39/32,587(34); 16/15,749(35)) of patients referred for cytogenetic testing because of developmental delay/congenital malformations/ASD $(34,35)$. We note that prior studies of the 7q11.23 duplication phenotype involve mainly pediatric patients. As the typical age of onset for psychosis in schizophrenia is in early adulthood, risk for psychosis in 7q11.23 duplication carriers is therefore not captured. Studies including adult patients are limited and omit psychiatric evaluation. For example, a 2009 study of twelve 7q11.23 duplication carrier probands with intellectual disability revealed seven were inherited; phenotypic descriptions of the parents neglected an evaluation for psychosis(32). Similarly, a 2011 study did not conduct psychiatric assessments on the eight adults identified with 7q11.23 duplication syndrome(33). We therefore may have uncovered a previously unrecognized aspect of the 
natural history of 7q11.23 duplication syndrome: adult risk for schizophrenia. This is reminiscent of 22q11 deletion syndrome, which is typically identified as a pediatric condition but carries substantial risk for schizophrenia in early adulthood. $(36,37)$. A longitudinal study of 7q11.23 duplication carriers would ideally assess the true risk for schizophrenia in this population.

It is possible we have ascertained adult individuals with autism rather than schizophrenia. However, the frequency of the 7q11.23 duplication in ASD patients is estimated at 4 in 3816 , or $0.1048 \%$ (23). Our study observes the 7q11.23 duplication 11 times in 14,573 individuals with schizophrenia. If we assume for the moment that all 11 duplication carriers have ASD, not SZ, and that this duplication is at the reported frequency of 0.1048 in the ASD population, that would imply $72 \%$ of all our SZ are actually misclassified ASD. Ascertainment procedures of SZ patients for the studies included herein are methodical, detailed, and comprehensive, and a $72 \%$ misclassification rate is highly unlikely. Assuming a more reasonable misclassification rate of $10 \%$ would imply 1 or 2 out of the 11 deletions are due to mischaracterized ASD. Since we observe the duplication 11 times, we conclude that any adult autism patients included in our sample cannot account for the enrichment we observe.

The 1.4 Mb region on chromosome 7q11.23 is flanked by segmental duplications (Figure 2), which are likely mediating non-allelic homologous recombination, giving rise to the duplication and reciprocal deletion. These rearrangements are typically de novo events: Sanders et al found that 4 out of 4 duplications were de novo(23); Girirajan et al found of 9 informative duplications, 5 were de novo (an additional 30 duplications in this study did not have parental DNAs available for inheritance testing)(34). The reciprocal deletion gives rise to the well-characterized Williams-Beuren syndrome (OMIM \#194050), first described in the early $1960 \mathrm{~s}(38,39)$. The duplication syndrome has only been described more recently. In 2005, a single patient with the duplication and severe language delay was described(40); later, description of the microduplication syndrome (OMIM \#609757) was expanded to include speech delay and other variable characteristics, such as ID, hypotonia, congenital heart defects, social interaction difficulties and, less often, epilepsy(32). More recently, de novo duplication of this region was linked to ASD, where hallmarks of the phenotype are diminished development of language and poor social communication $(23,41)$. In one of the largest and most comprehensive studies to date, Velleman and Mervis describe the cognitive and behavioral profile of 7q11.23 duplication syndrome derived from both literature reports and their own direct evaluation of 30 children with the duplication(33). They confirm both language delay and social anxiety in 7q11.23 duplication carriers, and find separation anxiety to feature prominently among these individuals $(33,42)$. In fact, these authors suggest that this separation anxiety, which can manifest with selective mutism, could resemble an autism phenotype(33). In a research setting the authors document social phenomena including eye contact, pleasurable engagement with a parent, imaginative play, and other reciprocal social interactions inconsistent with autism(33). While further study will be required to confirm or refute the autism-7q11.23 duplication relationship, our current data support a link between social anxiety, language delay, and the 7q11.23 duplication. Both SZ duplication carriers in the Ashkenazi sample report severe social anxiety and language delay (data in the Supplement). Similarly, a Bulgarian duplication carrier with SZ also had speech delay and is described as socially withdrawn(30). The mean age at onset of the $11 \mathrm{SZ}$ duplication carriers we report here is 20.7 ( $\mathrm{SD}=8.3$ years), however two cases had an onset in childhood (aged 7 and 8 years), suggesting that the age at onset could be quite early in a proportion of carriers. In light of the WBS phenotype, it is possible there may be a quantitative relationship between dosage of the 7q11.23 region and verbal and social skills, though more detailed phenotypic data will be necessary to investigate this hypothesis. 
Heterogeneity is a hallmark of schizophrenia. Among any population of SZ patients, there is a wide spectrum of differences in clinical presentation, course, and outcome. It has long been suspected that this spectrum reflects heterogeneity in underlying etiology, and current $\mathrm{CNV}$ data support this notion. In our sample, we note that the two unrelated patients in the AJ cohort had similar early childhood morbidity, including obsessions and compulsions, eating disorders, and language delay. Though more research is required, including detailed phenotypic studies and collection of retrospective data on duplication carriers, our findings nevertheless suggest the 7q11.23 region may define a specific subtype of SZ. Categorizing patients by etiologic variants like this may ultimately serve to reduce the vast heterogeneity seen in this disorder.

We find duplications at 7q11.23 in SZ cases at a frequency of $0.08 \%$, similar to the frequency of this duplication in ASD (0.1\%, four out of 3816 cases)(23). In two large samples of individuals referred for array CGH testing with a heterogeneous mixture of phenotypes, including congenital abnormalities, developmental delay, and intellectual disability, this duplication is seen in 39 out of 32,587 individuals (0.12\%) (34), and 16 of 15,749 individuals $(0.1 \%)$ (35), respectively. Thus, this duplication is seen with similar frequencies in patients with either SZ, ASD, or ID, echoing results from other CNVs associated with both SZ and ASD. Notably, thus far only a single duplication has been described in controls; most carriers are found in ID, autism, or SZ cohorts, underscoring the high pathogenicity of this duplication, which is similar to the $3 \mathrm{q} 29$ or $22 \mathrm{q} 11$ deletions( 7 , 14).

In summary, we find that the 7q11.23 duplication is a new risk factor for the development of SZ. This duplication shares the hallmarks of other SZ-associated CNVs: it is rare, has a large effect size, and is associated not only with schizophrenia, but also with autism and ID, supporting an etiologic link between these phenotypes. Future study incorporating comprehensive data collection will be required to understand the apparently different behavioral manifestations of the 7q11.23 deletion and duplication.

\section{Supplementary Material}

Refer to Web version on PubMed Central for supplementary material.

\section{Acknowledgments}

The authors wish to thank the individuals diagnosed with schizophrenia and their family members who participated in this research. Funding for this study was provided by NIH grants MH080129 and MH083722 to STW, and a NARSAD Young Investigator Award to JGM. Support was provided by MH077139 (PFS), the Stanley Center for Psychiatric Research at the Broad Institute from a grant from Stanley Medical Research Institute, the Karolinska Institutet, Karolinska University Hospital, the Swedish Research Council, ALF grant from Swedish County Council, and Söderström Königska Foundation. Funding support for the companion studies, Genome-Wide Association Study of Schizophrenia (GAIN) and Molecular Genetics of Schizophrenia - nonGAIN Sample (MGS_nonGAIN), was provided by Genomics Research Branch at NIMH and the genotyping and analysis of samples was provided through the Genetic Association Information Network (GAIN) and under the MGS U01s: MH79469 and MH79470. Assistance with data cleaning was provided by the National Center for Biotechnology Information. The MGS dataset(s) used for the analyses described in this manuscript were obtained from the database of Genotype and Phenotype (dbGaP) found at http://www.ncbi.nlm.nih.gov/gap through dbGaP accession numbers phs000021.v2.p1 (GAIN) and phs000167.v1.p1 (nonGAIN). Samples and associated phenotype data for the MGS GWAS study were collected under the following grants: NIMH Schizophrenia Genetics Initiative U01s: MH46276 (CR Cloninger), MH46289 (C Kaufmann), and MH46318 (MT Tsuang); and MGS Part 1 (MGS1) and Part 2 (MGS2) R01s: MH67257 (NG Buccola), MH59588 (BJ Mowry), MH59571 (PV Gejman), MH59565 (Robert Freedman), MH59587 (F Amin), MH60870 (WF Byerley), MH59566 (DW Black), MH59586 (JM Silverman), MH61675 (DF Levinson), and MH60879 (CR Cloninger). Further details of collection sites, individuals, and institutions may be found in data supplement Table 1 of(43) and at the study dbGaP pages. We wish to thank L. Ozelius (Mt. Sinai University) and M. Abreu (University of Miami) for contribution of AJ control samples. 
We also wish to thank the members of the The Molecular Genetics of Schizophrenia Consortium, which include: Douglas F. Levinson, M.D. (Stanford University), Jubao Duan, Ph.D. (NorthShore University HealthSystem Research Institute), Sang Oh (Stanford University), Kai Wang, Ph.D. (UCLA), Alan R. Sanders, M.D. (NorthShore University HealthSystem Research Institute), Jianxin Shi. Ph.D. (NCI), Nancy Zhang, Ph.D. (Stanford University), Bryan J. Mowry, M.D., F.R.A.N.Z.C.P. (Queensland Centre for Mental Health Research and Queensland Institute for Medical Research), Ann Olincy, M.D. (University of Colorado Denver), Farooq Amin, M.D. (Emory University), C. Robert Cloninger, M.D. (Washington University), Jeremy M. Silverman, M.D. (Mount Sinai School of Medicine), Nancy G. Buccola, A.P.R.N., B.C. (Louisiana State University Health Sciences Center), William F. Byerley, M.D. (UCSF), Donald W. Black, M.D. (University of Iowa Carver College of Medicine), Kenneth S. Kendler, M.D. (Virginia Commonwealth University), Robert Freedman, M.D. (University of Colorado Denver), Frank Dudbridge, Ph.D. (London School of Hygiene \& Tropical Medicine), Itsik Pe'er, Ph.D. (Columbia University), Hakon Hakonarson, M.D., Ph.D. (Children's Hospital of Philadelphia and University of Pennsylvania), Sarah E. Bergen, Ph.D. (Massachusetts General Hospital), Ayman H. Fanous, M.D. (Georgetown University School of Medicine), Peter A. Holmans, Ph.D. (Cardiff University) and Pablo V. Gejman, M.D. (NorthShore University HealthSystem Research Institute).

\section{References}

1. Gottesman, II.; Shields, J.; Hanson, DR. Schizophrenia, the epigenetic puzzle. Cambridge ; New York: Cambridge University Press; 1982.

2. Walsh T, McClellan JM, McCarthy SE, Addington AM, Pierce SB, Cooper GM, et al. Rare structural variants disrupt multiple genes in neurodevelopmental pathways in schizophrenia. Science. 2008; 320(5875):539-43. [PubMed: 18369103]

3. Consortium IS. Rare chromosomal deletions and duplications increase risk of schizophrenia. Nature. 2008; 455(7210):237-41. [PubMed: 18668038]

4. Kirov G, Grozeva D, Norton N, Ivanov D, Mantripragada KK, Holmans P, et al. Support for the involvement of large copy number variants in the pathogenesis of schizophrenia. Hum Mol Genet. 2009; 18(8):1497-503. [PubMed: 19181681]

5. Stefansson H, Rujescu D, Cichon S, Pietilainen OP, Ingason A, Steinberg S, et al. Large recurrent microdeletions associated with schizophrenia. Nature. 2008; 455(7210):232-6. [PubMed: 18668039]

6. Karayiorgou M, Morris MA, Morrow B, Shprintzen RJ, Goldberg R, Borrow J, et al. Schizophrenia susceptibility associated with interstitial deletions of chromosome 22q11. Proc Natl Acad Sci U S A. 1995; 92(17):7612-6. [PubMed: 7644464]

7. Levinson DF, Duan J, Oh S, Wang K, Sanders AR, Shi J, et al. Copy number variants in schizophrenia: confirmation of five previous findings and new evidence for 3q29 microdeletions and VIPR2 duplications. Am J Psychiatry. 2011; 168(3):302-16. [PubMed: 21285140]

8. Mulle JG, Dodd AF, McGrath JA, Wolyniec PS, Mitchell AA, Shetty AC, et al. Microdeletions of 3q29 confer high risk for schizophrenia. Am J Hum Genet. 2010; 87(2):229-36. [PubMed: 20691406]

9. Kirov G, Rujescu D, Ingason A, Collier DA, O’Donovan MC, Owen MJ. Neurexin 1 (NRXN1) deletions in schizophrenia. Schizophr Bull. 2009; 35(5):851-4. [PubMed: 19675094]

10. Kirov G, Gumus D, Chen W, Norton N, Georgieva L, Sari M, et al. Comparative genome hybridization suggests a role for NRXN1 and APBA2 in schizophrenia. Hum Mol Genet. 2008; 17(3):458-65. [PubMed: 17989066]

11. Friedman JI, Vrijenhoek T, Markx S, Janssen IM, van der Vliet WA, Faas BH, et al. CNTNAP2 gene dosage variation is associated with schizophrenia and epilepsy. Mol Psychiatry. 2008; 13(3): 261-6. [PubMed: 17646849]

12. McCarthy SE, Makarov V, Kirov G, Addington AM, McClellan J, Yoon S, et al. Microduplications of 16p11.2 are associated with schizophrenia. Nat Genet. 2009; 41(11):1223-7. [PubMed: 19855392]

13. Vacic V, McCarthy S, Malhotra D, Murray F, Chou HH, Peoples A, et al. Duplications of the neuropeptide receptor gene VIPR2 confer significant risk for schizophrenia. Nature. 2011; 471(7339):499-503. [PubMed: 21346763]

14. Grozeva D, Conrad DF, Barnes CP, Hurles M, Owen MJ, O’Donovan MC, et al. Independent estimation of the frequency of rare CNVs in the UK population confirms their role in schizophrenia. Schizophr Res. 2012; 135(1-3):1-7. [PubMed: 22130109] 
15. ISC. Rare chromosomal deletions and duplications increase risk of schizophrenia. Nature. 2008; 455(7210):237-41. [PubMed: 18668038]

16. Moreno-De-Luca D, Mulle JG, Kaminsky EB, Sanders SJ, Myers SM, Adam MP, et al. Deletion $17 q 12$ is a recurrent copy number variant that confers high risk of autism and schizophrenia. Am J Hum Genet. 2010; 87(5):618-30. [PubMed: 21055719]

17. Rujescu D, Ingason A, Cichon S, Pietilainen OP, Barnes MR, Toulopoulou T, et al. Disruption of the neurexin 1 gene is associated with schizophrenia. Hum Mol Genet. 2009; 18(5):988-96. [PubMed: 18945720]

18. Mefford HC, Sharp AJ, Baker C, Itsara A, Jiang Z, Buysse K, et al. Recurrent rearrangements of chromosome 1q21.1 and variable pediatric phenotypes. N Engl J Med. 2008; 359(16):1685-99. [PubMed: 18784092]

19. Mefford HC, Clauin S, Sharp AJ, Moller RS, Ullmann R, Kapur R, et al. Recurrent reciprocal genomic rearrangements of 17q12 are associated with renal disease, diabetes, and epilepsy. Am J Hum Genet. 2007; 81(5):1057-69. [PubMed: 17924346]

20. Crespi B, Stead P, Elliot M. Evolution in health and medicine Sackler colloquium: Comparative genomics of autism and schizophrenia. Proc Natl Acad Sci U S A. 2010; 107(Suppl 1):1736-41. [PubMed: 19955444]

21. Girirajan S, Rosenfeld JA, Coe BP, Parikh S, Friedman N, Goldstein A, et al. Phenotypic heterogeneity of genomic disorders and rare copy-number variants. N Engl J Med. 2012; 367(14): 1321-31. [PubMed: 22970919]

22. Owen MJ. Intellectual disability and major psychiatric disorders: a continuum of neurodevelopmental causality. Br J Psychiatry. 2012; 200:268-9. [PubMed: 22474230]

23. Sanders SJ, Ercan-Sencicek AG, Hus V, Luo R, Murtha MT, Moreno-De-Luca D, et al. Multiple recurrent de novo $\mathrm{CNVs}$, including duplications of the 7q11.23 Williams syndrome region, are strongly associated with autism. Neuron. 2011; 70(5):863-85. [PubMed: 21658581]

24. Fallin MD, Lasseter VK, Avramopoulos D, Nicodemus KK, Wolyniec PS, McGrath JA, et al. Bipolar I disorder and schizophrenia: a 440-single-nucleotide polymorphism screen of 64 candidate genes among Ashkenazi Jewish case-parent trios. Am J Hum Genet. 2005; 77(6):91836. [PubMed: 16380905]

25. Hupe P, Stransky N, Thiery JP, Radvanyi F, Barillot E. Analysis of array CGH data: from signal ratio to gain and loss of DNA regions. Bioinformatics. 2004; 20(18):3413-22. [PubMed: 15381628]

26. Pique-Regi R, Caceres A, Gonzalez JR. R-Gada: a fast and flexible pipeline for copy number analysis in association studies. BMC Bioinformatics. 2010; 11:380. [PubMed: 20637081]

27. Satten G, Allen A, Ikeda M, Mulle JG, Warren ST. Robust regression analysis of copy number variation data based on a univariate score. 2012 Submitted.

28. Agresti, A. Categorical Data Analysis. New York: Wiley; 1990.

29. Agresti, A. Categorical Data Analysis: Second Edition. New York: Wiley; 2002.

30. Kirov G, Pocklington AJ, Holmans P, Ivanov D, Ikeda M, Ruderfer D, et al. De novo CNV analysis implicates specific abnormalities of postsynaptic signalling complexes in the pathogenesis of schizophrenia. Mol Psychiatry. 2012; 17(2):142-53. [PubMed: 22083728]

31. Stewart LR, Hall AL, Kang SH, Shaw CA, Beaudet AL. High frequency of known copy number abnormalities and maternal duplication 15q11-q13 in patients with combined schizophrenia and epilepsy. BMC Med Genet. 2011; 12:154. [PubMed: 22118685]

32. Van der Aa N, Rooms L, Vandeweyer G, van den Ende J, Reyniers E, Fichera M, et al. Fourteen new cases contribute to the characterization of the $7 \mathrm{q} 11.23$ microduplication syndrome. Eur J Med Genet. 2009; 52(2-3):94-100. [PubMed: 19249392]

33. Velleman SL, Mervis CB. Children with 7q11.23 Duplication Syndrome: Speech, Language, Cognitive, and Behavioral Characteristics and their Implications for Intervention. Perspectives on language learning and education. 2011; 18(3):108-16. [PubMed: 22754604]

34. Girirajan S, Rosenfeld JA, Coe BP, Parikh S, Friedman N, Goldstein A, et al. Phenotypic Heterogeneity of Genomic Disorders and Rare Copy-Number Variants. N Engl J Med. 2012

35. Kaminsky EB, Kaul V, Paschall J, Church DM, Bunke B, Kunig D, et al. An evidence-based approach to establish the functional and clinical significance of copy number variants in 
intellectual and developmental disabilities. Genetics in medicine : official journal of the American College of Medical Genetics. 2011; 13(9):777-84. [PubMed: 21844811]

36. Pulver AE, Nestadt G, Goldberg R, Shprintzen RJ, Lamacz M, Wolyniec PS, et al. Psychotic illness in patients diagnosed with velo-cardio-facial syndrome and their relatives. J Nerv Ment Dis. 1994; 182(8):476-8. [PubMed: 8040660]

37. Murphy KC, Jones LA, Owen MJ. High rates of schizophrenia in adults with velo-cardio-facial syndrome. Arch Gen Psychiatry. 1999; 56(10):940-5. [PubMed: 10530637]

38. Williams JC, Barratt-Boyes BG, Lowe JB. Supravalvular aortic stenosis. Circulation. 1961; 24:1311-8. [PubMed: 14007182]

39. Beuren AJ, Apitz J, Harmjanz D. Supravalvular aortic stenosis in association with mental retardation and a certain facial appearance. Circulation. 1962; 26:1235-40. [PubMed: 13967885]

40. Somerville MJ, Mervis CB, Young EJ, Seo EJ, del Campo M, Bamforth S, et al. Severe expressive-language delay related to duplication of the Williams-Beuren locus. N Engl J Med. 2005; 353(16):1694-701. [PubMed: 16236740]

41. American Psychiatric Association., American Psychiatric Association. Diagnostic and statistical manual of mental disorders : DSM-IV. 4. Washington, DC: American Psychiatric Association; 1994. Task Force on DSM-IV.

42. Mervis CB, Dida J, Lam E, Crawford-Zelli NA, Young EJ, Henderson DR, et al. Duplication of GTF2I results in separation anxiety in mice and humans. Am J Hum Genet. 2012; 90(6):1064-70. [PubMed: 22578324]

43. Sanders AR, Duan J, Levinson DF, Shi J, He D, Hou C, et al. No significant association of 14 candidate genes with schizophrenia in a large European ancestry sample: implications for psychiatric genetics. Am J Psychiatry. 2008; 165(4):497-506. [PubMed: 18198266]

44. Shi J, Levinson DF, Duan J, Sanders AR, Zheng Y, Pe'er I, et al. Common variants on chromosome 6p22.1 are associated with schizophrenia. Nature. 2009; 460(7256):753-7. [PubMed: 19571809]

45. Ikeda M, Aleksic B, Kirov G, Kinoshita Y, Yamanouchi Y, Kitajima T, et al. Copy number variation in schizophrenia in the Japanese population. Biol Psychiatry. 2010; 67(3):283-6. [PubMed: 19880096]

46. Sullivan PF, Lin D, Tzeng JY, van den Oord E, Perkins D, Stroup TS, et al. Genomewide association for schizophrenia in the CATIE study: results of stage 1. Mol Psychiatry. 2008; 13(6): 570-84. [PubMed: 18347602]

47. Bergen SE, O’Dushlaine CT, Ripke S, Lee PH, Ruderfer D, Akterin S, et al. Genome-wide association study in a Swedish population yields support for greater CNV and MHC involvement in schizophrenia compared to bipolar disorder. Mol Psychiatry. 2012 

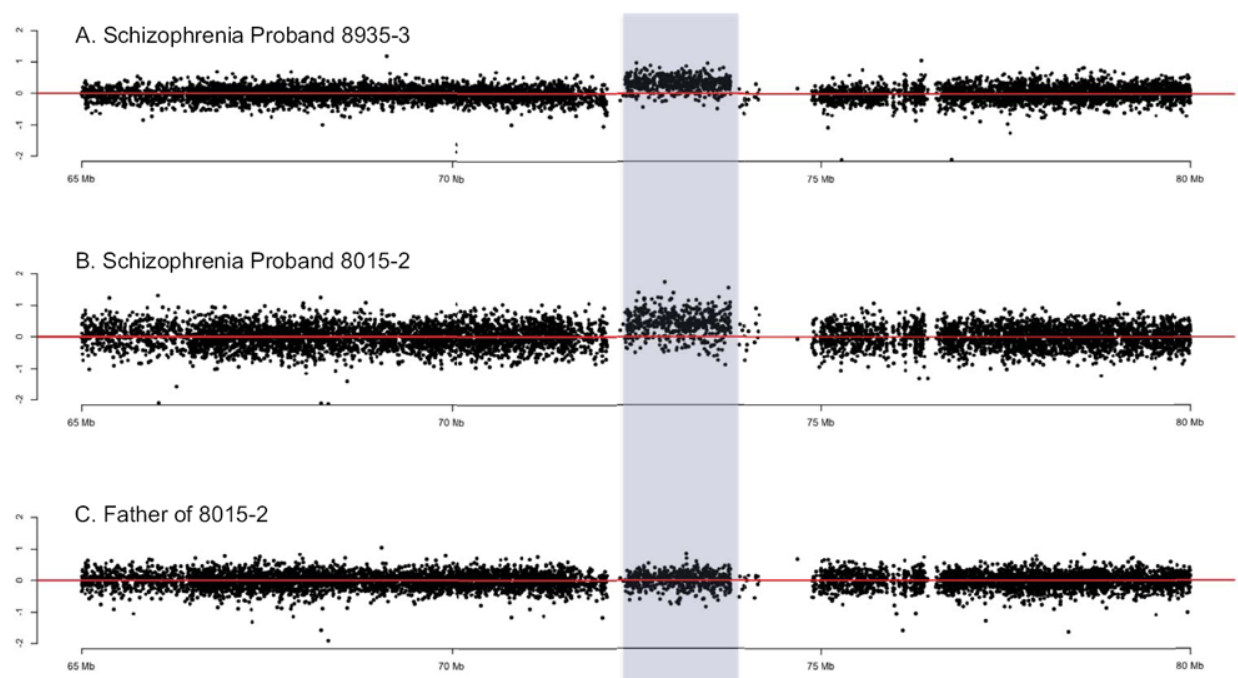

D. Mother of 8015-2

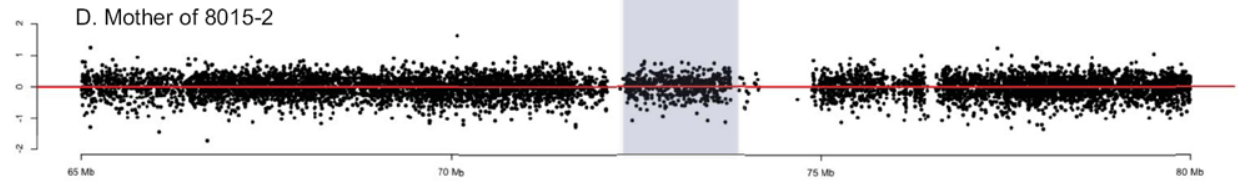

Figure 1.

Raw $\log (2)$ ratio data are shown for $15 \mathrm{Mb}$ on chromosome $7 \mathrm{q}(65 \mathrm{Mb}-80 \mathrm{Mb}$ ) flanking the duplication region, for two unrelated AJ schizophrenia probands carrying the 7q11.23 duplication (panels A and B). Shading indicates the duplicated region. The parents of SZ proband 8015-2, who do not carry the duplication, are also shown (panels C and D). 


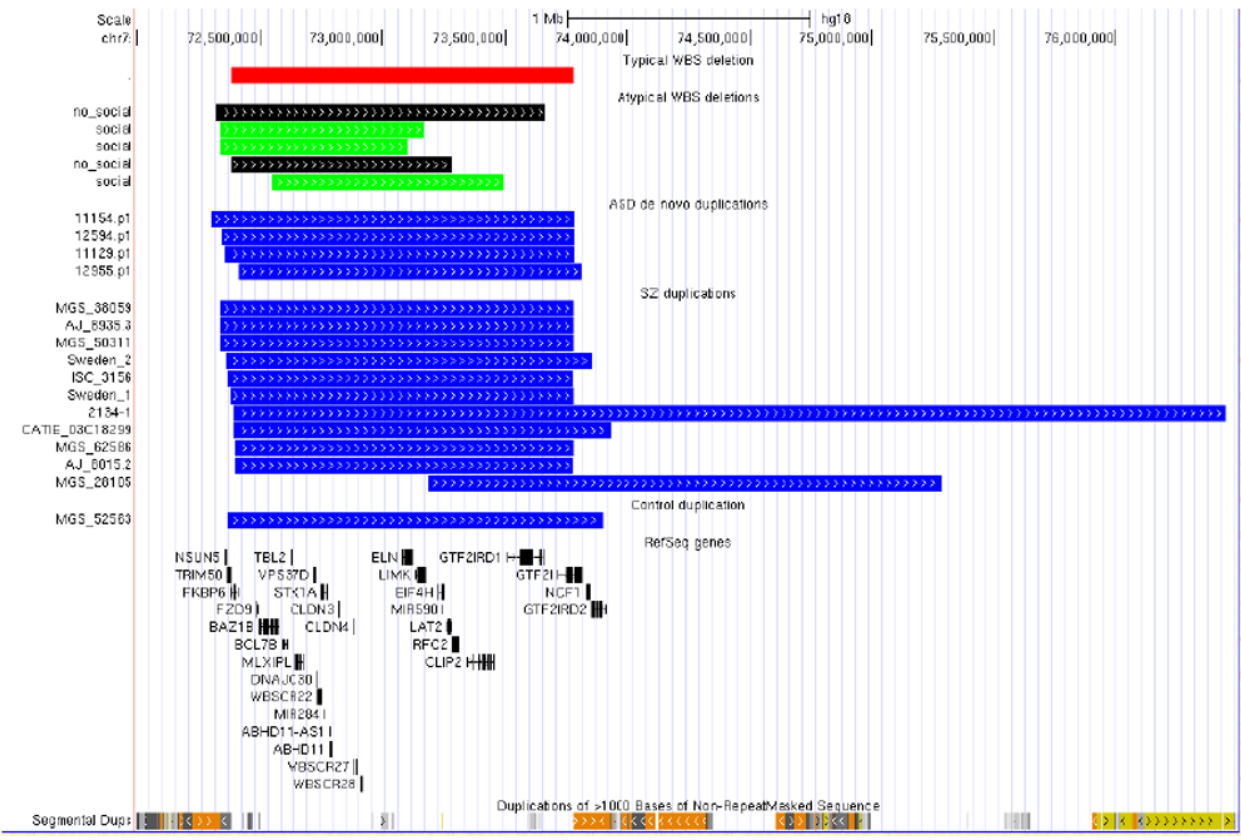

Figure 2.

Eleven duplications found in SZ patients are shown against the context of previously reported events at the 7q11.23 locus, including typical and atypical WBD deletions (with highly social or not highly social phenotypes noted), de novo duplications in ASD patients, and genes in the WBS region. 


\section{Table 1}

Excess rare variants in 554 SZ cases vs 1014 controls

\begin{tabular}{|l|l|l|l|l|}
\hline Type of variant & $\begin{array}{l}\text { Number of cases with at least 1 } \\
\text { variant }\end{array}$ & $\begin{array}{l}\text { Number of controls with at least 1 } \\
\text { variant }\end{array}$ & p-value & Odds Ratio (95\% CI) \\
\hline Rare CNV > $500 \mathrm{~kb}$ & $31(5.6 \%)$ & $24(2.4 \%)$ & 0.0013 & $2.44(1.37-4.4)$ \\
\hline Deletions > $500 \mathrm{~kb}$ & $13(2.3 \%)$ & $8(0.8 \%)$ & 0.018 & $3.02(1.15-8.46)$ \\
\hline Duplications > $500 \mathrm{~kb}$ & $18(3.2 \%)$ & $16(1.6 \%)$ & 0.044 & $2.09(1.00-4.43)$ \\
\hline Rare CNV > $1 \mathrm{Mb}$ & $19(3.4 \%)$ & $10(1 \%)$ & 0.0012 & $3.56(1.56-8.64)$ \\
\hline Deletions > $1 \mathrm{Mb}$ & $11(2 \%)$ & $4(0.4 \%)$ & 0.0043 & $5.11(1.51-22.1)$ \\
\hline Duplications $>1 \mathrm{Mb}$ & $8(1.4 \%)$ & $6(0.8 \%)$ & 0.097 & $2.46(0.74-8.65)$ \\
\hline
\end{tabular}

All odds ratios and p-values calculated using a two-sided Fisher's exact test in R. 
Table 2

Meta-Analysis of the 7q11.23 duplication in SZ cohorts

\begin{tabular}{|l|l|l|l|l|}
\hline Sample & Cases & Case 7q11.23 Duplications & Controls & Control 7q11.23 Duplications \\
\hline Ashkenazi & 554 & $2^{*}$ & 1014 & 0 \\
\hline MGS(44) & 3945 & 4 & 3611 & 1 \\
\hline ISC(3) & 3063 & 1 & 3181 & 0 \\
\hline Bulgarian Trios(30) & 662 & $1^{* *}$ & 638 & 0 \\
\hline Japanese(45) & 575 & 0 & 564 & 0 \\
\hline UK(4, 14) & 471 & 0 & 13,036 & 0 \\
\hline CATIE(46) & 738 & 1 & 289 & 0 \\
\hline Sweden(47) & 4379 & 2 & 5806 & 0 \\
\hline Totals & 14,387 & 11 & 28,139 & 1 \\
\hline Raw OR & 21.52 (95\% CI: 3.13-922.6, p-value 5.5 $\left.510^{-5}\right)$ & \\
\hline MH-corrected OR & $10.78(95 \%$ CI: $1.46-79.62, \mathrm{p}$-value 0.007$)$ & \\
\hline \\
one de novo event; \\
** \\
de novo event
\end{tabular}

